\title{
Flash visual evoked potentials are unreliable as markers of ICP due to high variability in normal subjects
}

\author{
Linnea Andersson • Johanna Sjölund • Josefin Nilsson
}

Received: 28 August 2011 / Published online: 30 September 2011

(C) Springer-Verlag 2011

Invasive ICP monitoring is a standard adjunct in modern treatment of head injury with brain edema and other conditions with intracranial hypertension. Along with continuous EEG and evoked potential monitoring, it enables us to detect and to react to the brain function abnormalities at a reversible stage. There is currently no reliable method of non-invasive ICP measurement.

This well-done laboratory research was not designed to study the relation between the two variables stated in the title (FVEP and ICP), but to get normative data from test-retest FVEP measurements in awake healthy volunteers. High variability found in awake normal subjects makes questionable the results of clinical trials on non-invasive ICP measurement by using FVEP in sedated/comatose patients. Intracranial hypertension profoundly influences metabolically coupled conduction function of the brain and cortical evoked responses, as well as some anesthetics. The linear positive correlation between latencies of negative FVEP components and ICP values found by many authors raise questions on conceptual, methodological and anesthesiological differences in these researches, diversity of the intracranial pathologies included and possibly subcortical origin of some FVEP components. At least, preliminary variability study of their method in uniform experimental settings should be performed before clinical trials, as a basic requirement.

Roman Bosnjak

Ljubljana, Slovenia

1. Benedicic M, Bosnjak R (2011) Intraoperative monitoring of the visual function using cortical potentials after electrical epidural stimulation of the optic nerve. Acta Neurochir (Wien) 153(10):1919-1927

Conflicts of interest None.

\footnotetext{
Support Author J.N. was supported by grants from ALF-medel (Agreement concerning research and education of doctors) no ALFGBG-146731 and The Swedish Society of Medicine grant no 2009-22093.

L. Andersson · J. Sjölund · J. Nilsson

Department of Clinical Neuroscience, Institute of Neuroscience and Physiology,

The Sahlgrenska Academy at the University of Gothenburg,

Göteborg, Sweden

J. Nilsson ( $\square)$

Department of Clinical Neurophysiology,

Sahlgrenska University Hospital,

Blå stråket 7-3 tr,

41345 Göteborg, Sweden

e-mail: josefin.nilsson@neuro.gu.se
} 